\title{
Restoring the Co Magnetic Moments at Interfacial Co-Porphyrin Arrays by Site-Selective Uptake of Iron
}

\author{
Saranyan Vijayaraghavan, ${ }^{\dagger}$ Willi Auwärter, ${ }^{*,+}$ David Ecija, ${ }^{\dagger, \nabla}$ Knud Seufert, $^{\dagger, \otimes}{ }^{\text {Stefano Rusponi, }}{ }^{*}$ \\ Torsten Houwaart, ${ }^{\S}$ Philippe Sautet, ${ }^{\S}$ Marie-Laure Bocquet, ${ }^{\S}{ }^{`}$ Pardeep Thakur, ${ }^{\perp}$ Sebastian Stepanow," \\ Uta Schlickum, ${ }^{\neq, \#}$ Markus Etzkorn, ${ }^{\neq, \#}$ Harald Brune, ${ }^{\ddagger}$ and Johannes V. Barth ${ }^{\dagger}$ \\ ${ }^{\dagger}$ Physik-Department E20, Technische Universität München, D-85748 Garching, Germany, ${ }^{\ddagger}$ Institute of Condensed Matter Physics (ICMP), Ecole Polytechnique \\ Fédérale de Lausanne (EPFL), CH-1015 Lausanne, Switzerland, ${ }^{\S}$ Université de Lyon, CNRS, Ecole Normale Supérieure de Lyon, Laboratoire de Chimie, F-69364 Cedex \\ 07 Lyon, France, ${ }^{\perp}$ European Synchrotron Radiation Facility (ESRF), B.P. 220, Grenoble Cedex F-38043, France, "Department of Materials, ETH Zürich, Hönggerbergring 64, \\ CH-8093 Zürich, Switzerland, and "Max Planck Institut für Festkörperforschung, D-70569 Stuttgart, Germany. P Present address (D.E.): IMDEA Nanoscience, E-28049 \\ Madrid, Spain. ${ }^{\otimes}$ Present address (K.S.): Institut für Chemie, Karl-Franzens-Universität Graz, A-8010 Graz, Austria. "Present address (M.-L.B.): Ecole Normale Supérieure-PSL \\ Research University, Département de Chimie, Sorbonne Universités - UPMC Univ. Paris 06, CNRS UMR 8640 PASTEUR, F-75005 Paris, France.
}

\begin{abstract}
Magnetochemistry recently emerged as a promising approach to control addressable spin arrays on surfaces. Here we report on the binding, spatial ordering, and magnetic properties of Fe on a highly regular Co-tetraphenylporphyrin (Co-TPP) template and highlight how the Fe controls the magnetism of the Co centers. As evidenced by scanning tunneling microscopy (STM) single Fe atoms attach to the saddle-shape conformers site-selectively in a unique coordination environment offered through a heptamer defined by the $\mathrm{Co}-\mathrm{N}-\mathrm{C}-\mathrm{C}-\mathrm{C}-\mathrm{N}$ cyclic subunit. While the

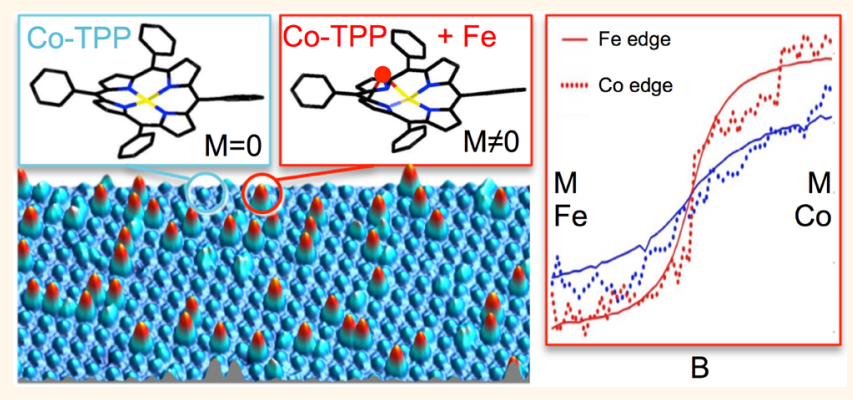
magnetic moment of $\mathrm{Co}_{0}$ is quenched for bare Co-TPP/Ag(111), the Fe presence revives it. Our X-ray magnetic circular dichroism (XMCD) experiments, complemented by density functional theory (DFT) calculations, evidence a ferromagnetic coupling between the Fe and the Co center concomitant with a complex charge redistribution involving the porphyrin ligand. Thus, we demonstrate an unusual metalloporphyrin coordination geometry that opens pathways to spatially order and engineer magnetic moments in surface-based nanostructures.
\end{abstract}

KEYWORDS: magnetochemistry $\cdot$ magnetic moment $\cdot$ metalloporphyrin $\cdot$ template $\cdot$ scanning tunneling microscopy (STM) · $\mathrm{X}$-ray magnetic circular dichroism (XMCD) · density functional theory (DFT)

$\mathrm{T}$ he fabrication of addressable, surfacebased arrays comprising single atomic spins and magnetic moments is a key to comprehensively understand magnetic phenomena on the nanoscale, ${ }^{1-3}$ and to realize potential sensing, information storage, spintronic or quantum computing devices. ${ }^{4-7}$ Recently, the bottom-up assembly of metal-organic magnetic layers and architectures on surfaces has attracted considerable interest, given the versatility of molecular building blocks combined with metal centers featuring magnetic moments. ${ }^{8-10}$ Prominent results include multicomponent two-dimensional metalorganic networks ${ }^{11,12}$ or dense-packed arrays of metal-organic complexes, ${ }^{2,13}$ where unpaired $d$ - or f-electrons contribute to the magnetic moment for transition metal and lanthanide centers, respectively. This selfassembly approach guarantees an exquisite control on the positions and the local coordination sphere of the magnetic centers, comparable to the precise atom positioning achieved by serial manipulation procedures based on low-temperature scanning tunneling microscopy (STM), ${ }^{14,15}$ which however are not applicable for large-scale patterning of substrates, a frequent criterion for applications.

Commonly, the interaction of molecules and metal centers with the supporting surface affects their electronic and magnetic properties, and tetrapyrrole species have been frequently employed for exemplary investigations in this context. ${ }^{16}$ While this *Address correspondence to
wau@tum.de.

Received for review June 16, 2014 and accepted March 27, 2015.

Published online April 09, 2015 10.1021/nn507346x

C 2015 American Chemical Society 
interaction can be beneficial, e.g., on ferromagnetic substrates to induce magnetic order, ${ }^{17-19}$ it frequently evokes an adsorption-induced reduction or even quenching of intrinsic magnetic moments. ${ }^{20-24}$ In this context, recent studies have introduced the concept of confining metal atoms on self-assembled organic layers. ${ }^{25-27}$ This approach aims at ordering metallic atoms and clusters while at the same time counterbalancing the substrate influence; i.e., the organic layers are envisioned to act as template enabling the bottom-up assembly of regular and tunable arrays of magnetic moments, with a control on the magnetic coupling. From a different perspective, a site-specific confinement of adsorbates on (metal-)organic layers provides suitable model systems to explore donoracceptor systems on a single atom level ${ }^{28}$ and to tailor the magnetic properties of surface-anchored supramolecular architectures by atomic doping. ${ }^{29-31}$ Similarly, the use of gaseous ligands as nitric oxide, ${ }^{32-34}$ ammonia $^{13,35}$ or oxygen ${ }^{36}$ binding reversibly to metal-organic networks or adsorbed tetrapyrrole systems opened pathways to control and switch spin states of metal-centers, following the so-called onsurface magnetochemistry approach. ${ }^{13,33-36}$

Here, we employ a highly regular tetragonal metalorganic template with a periodicity of $1.4 \mathrm{~nm}$, namely a Co-tetraphenylporphyrin (Co-TPP) layer on $\mathrm{Ag}(111)$, guiding the positioning of individual $\mathrm{Fe}$ atoms and clusters upon exposure to an atomic beam of Fe in an ultrahigh vacuum environment. The charge redistribution in the metal-organic film by Fe induces a magnetic moment in Co, which was quenched in the bare Co-TPP layer on Ag(111). This behavior bases on a ferromagnetic coupling between the $\mathrm{Fe}$ and the $\mathrm{Co}$ center and is markedly different from the reduction of magnetic moments upon interaction with metal atoms in supports exemplified by the quenching of the Co spin in Co-Pc on a Fe surface. ${ }^{22}$ While it is well-known that transition metal centers in surface-anchored porphyrins provide an active site for ligation of gaseous adducts, ${ }^{33,37,38}$ the comprehensive characterization of their interaction with individual magnetic species has not been addressed. Furthermore, several reports exist, where systematic studies demonstrate ferromagnetic coupling between adlayers incorporating metalloporphyrins and underlying ultrathin magnetic films. ${ }^{17-19}$ To this end frequently the combination of XMCD measurements and DFT simulations proved instrumental. However, it is an open question how such systems behave in the extreme limit of single- to fewatom concentrations (i.e., monomers, dimers, clusters etc.) of magnetic elements and how the latter would interact with the magnetically active sites. Accordingly, a surface coordination chemistry approach ${ }^{39}$ is an important complement to the extensively studied magnetic interactions and switching phenomena of tetrapyrrole species contacting metallic nonmagnetic or magnetic layers, ${ }^{17-19,21-23}$ and their magnetochemistry upon ligation of small molecular species..$^{33-35}$ Moreover, it provides access to heterometal bonding at metalloporphyrins ${ }^{40,41}$ while enlarging scope and understanding of metalloporphyrins and other tetrapyrrole units with unusual geometries. ${ }^{42-44}$

Our combined experimental and theoretical efforts thus clarify the magnetic, electronic and structural properties of Fe on Co-TPP complexes with a unique coordination environment. These insights open novel avenues to engineer magnetic properties of interfacial coordination architectures employing novel binding motifs for individual transition metal atoms at metalloporphyrins and related species.

\section{RESULTS AND DISCUSSION}

Characterization of the Co-TPP Template. Figure 1 a shows an STM image of a Co-TPP array formed on $\mathrm{Ag}(111)$ after deposition at a sample temperature of $340 \mathrm{~K}$. The molecules assemble in extended and highly regular dense-packed domains with square symmetry and a periodicity of $14.1 \pm 0.2 \AA$. In agreement with earlier reports, six different orientations of the Co-TPP domains are observed. ${ }^{45} \mathrm{~A}$ bright central protrusion, originating from $\mathrm{Co}$ atomic states, dominates the appearance of Co-TPP/Ag(111) at low bias voltages (Figure $1 \mathrm{a},-0.4 \mathrm{~V}) .^{46}$ At higher negative bias voltages $\left(V_{\mathrm{b}}>-0.5 \mathrm{eV}\right)$, submolecular resolution evidence a 2-fold symmetry of the Co-TPP macrocycle (Figure $1 \mathrm{~b}$ ). As detailed in a previous study, this contrast is related to an adsorption induced saddle-shape deformation of Co-TPP, where the so-called $\alpha$-pyr axis dominates the appearance (cf. Figure $2 b$ ). ${ }^{45}$ Whereas the electronic structure of the Co-TPP/Ag(111) interface is well characterized, $^{37,45,47-49}$ the magnetic properties are addressed in this report for the first time. The Co-edge X-ray absorption spectra (XAS) for circular (Figure $1 \mathrm{c}-\mathrm{e}$ ) and linear (Figure 1f) polarized light were measured for the Co-TPP layer with perpendicular and grazing incidences, respectively (see inset in Figure 1c). In particular the $L_{3}$ absorption edge displays a clear fine structure indicating that the Co hybridization with the substrate is rather weak. The X-ray magnetic circular dichroism (XMCD) signal reveals no sign of dichroism within the noise (Figure 1e). The small dip visible is centered at the energy of maximum slope of the $\mathrm{L}_{3}$-edge and therefore can be attributed to an artifact resulting from errors in the energy alignment of the two circular polarizations. The absence of dichroism directly translates to zero net magnetization of the Co-center within the Co-TPP network. Specifically, even when considering the dip-like structure as a dichroic signal, from a sum rule analysis ${ }^{50,51}$ we can determine an upper limit of the magnetic moment in the Co center of $0.01 \mu_{\mathrm{B}}$. Thus, the Co moment is quenched in the first layer of Co-TPP adsorbed on $\mathrm{Ag}(111)$. By contrast, the detailed study of Co-TPP 

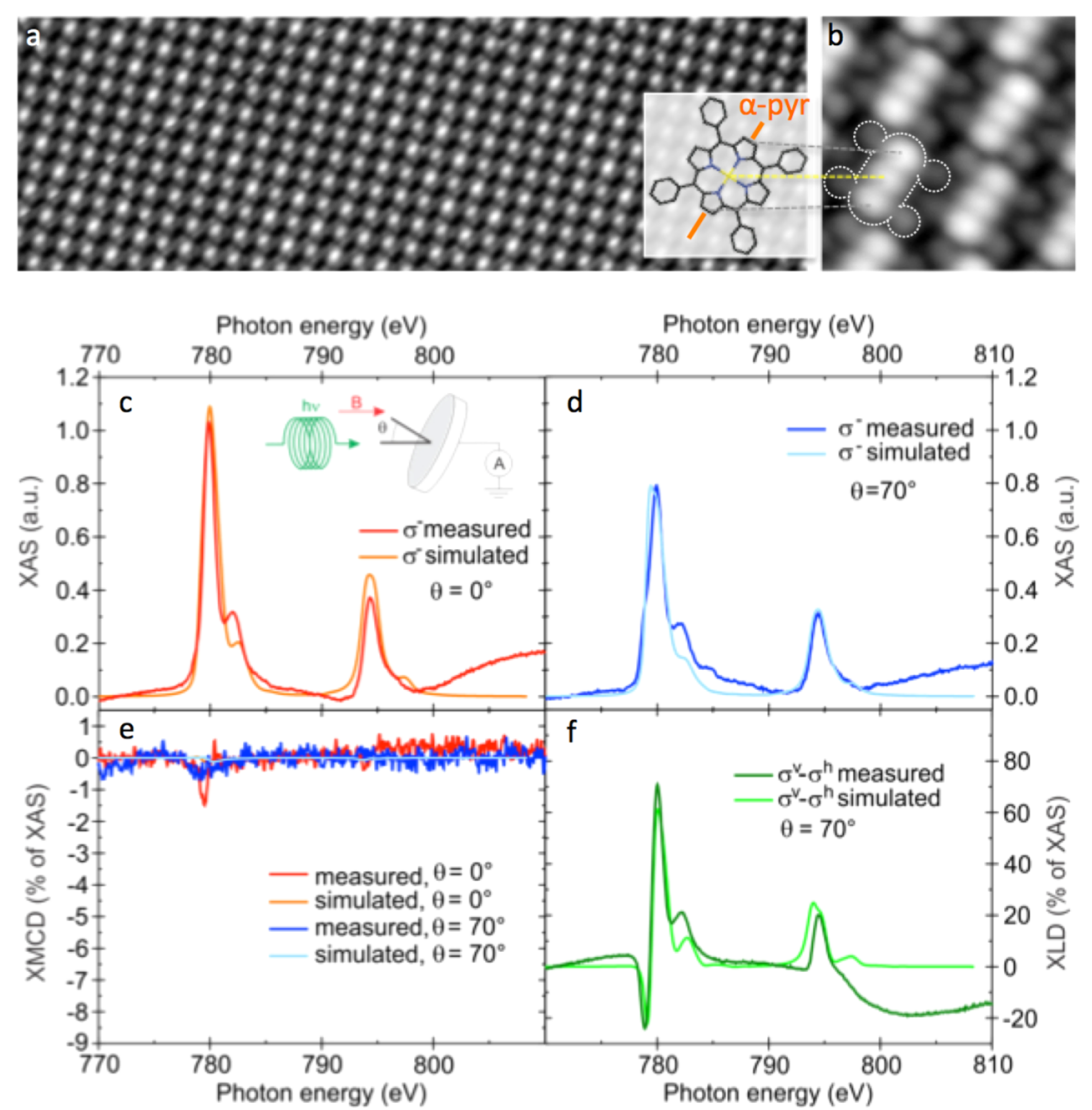

Figure 1. (a) STM image representing a regular Co-TPP array on $\mathrm{Ag}(111)\left(I=97 \mathrm{pA}, V_{\mathrm{b}}=-0.4 \mathrm{~V}, 443 \AA \times 143 \AA\right.$ ). (b) Highresolution image evidencing a 2 -fold symmetry of the Co-TPP macrocycle $\left(I=0.12 \mathrm{nA}, V_{\mathrm{b}}=-0.9 \mathrm{~V}, 20 \AA \times 20 \AA\right)$. (c-f) XAS measured on the bare monolayer of $\mathrm{Co}$-TPP on $\mathrm{Ag}(111)$ together with the fits obtained by the multiplet calculation. (c) XAS for $\sigma^{-}$and $\theta=0^{\circ}$. The inset shows the measuring geometry. (d) XAS for $\sigma^{-}$and $\theta=70^{\circ}$. (e) Vanishing XMCD $\left(\sigma^{+}-\sigma^{-}\right)$for $\theta=$ $0^{\circ}$ and $70^{\circ}$. (f) XLD $\left(\sigma^{\mathrm{v}}-\sigma^{\mathrm{h}}\right)$ for $\theta=70^{\circ}$. All spectra were measured at $T=8 \mathrm{~K}$ in magnetic fields of $B=5 \mathrm{~T}$ (c-e) and $B=0.2 \mathrm{~T}$ (f). In (c) and (d), the Ag background and a step function to compensate nonresonant absorption have been subtracted from the data. All X-ray absorption spectra have been normalized to the absorption intensity of the $\mathrm{L}_{3}$-edge maximum at $\theta=0^{\circ}$. Because of the small Co signal compared to the Ag background, residual modulations from $\mathrm{Ag}$ are visible in the spectra. This does not affect the Co XMCD spectrum as contributions of $\mathrm{Ag}$ cancel out. In contrast the Ag surface leads to a finite XLD of the background signal visible in (f); however, the structure of the Co L edges can be clearly determined.

and closely related metallophtalocyanines on ferromagnetic substrates revealed a strikingly different behavior. ${ }^{13,32}$ Without hybridization to a substrate, $e$. g., in gas phase or on graphene-passivated surfaces, the Co-center in tetrapyrrole units has a $d^{7}$-ground state and a low spin configuration $(S=1 / 2)^{52-54}$ with a magnetic moment of about $1.1 \mu_{\mathrm{B}}{ }^{55}$ The pronounced $X$-ray linear dichroism (XLD) measured at grazing incidence (Figure 1f) reflects the inhomogeneous charge distribution within the Co $3 d$ in- and out-of-plane orbitals (vide infra).

In order to rationalize the data we have performed DFT and ligand field multiplet calculations. For the latter we used the same code as described in ref 56. In these calculations we varied the charge transfer and crystal field parameters acting on the Co-center to fit the XAS data. For simplicity, we assumed the Co center environment to have 4-fold $\left(D_{4 h}\right)$ symmetry; i.e., the saddle shape deformation discussed above was neglected. The resulting simulated XAS, XMCD and XLD spectra are plotted along with the experimental data in Figure 1c-f. Good agreement between the experimental data and the multiplet calculations can be reached using high crystal field parameters $\left(10 D_{\mathrm{q}}=\right.$ $2.5 \mathrm{eV}, D_{\mathrm{s}}=0.38 \mathrm{eV}, D_{\mathrm{t}}=0.18 \mathrm{eV}$ ). A partial electron transfer from the substrate via the $d_{z^{2}}$-orbitals has to be included to achieve the above shown agreement, resulting in a new $d^{7}+d^{8}$ ground state (with a total of 7.25 electrons). The finite mixing with the low spin $\mathrm{d}^{8}$-states with $S=0$ forces singlet formation also in the $d^{7}$ configuration and results in a total quenching of the magnetic moment. The quenching of the spin moment follows the same arguments presented for CoPc on $\mathrm{Au}(111) .{ }^{56}$ Here, the Co ion assumes a $d^{7}$ configuration, and the substrate is modeled as a state with one electron. The substrate electron is allowed to hop onto the out-of-plane $d_{z^{2}}$ orbital of the Co ion thereby increasing the valence to $d^{8}$. This dynamical hybridization leads to the configuration mixing of the $d^{7} E$ and $d^{8}$ manifolds, where $E$ denotes the substrate electron. The crystal field potential imposed by the porphyrin ligand splits the energy levels of the $d^{7}$ and $d^{8}$ multiplets. Both 

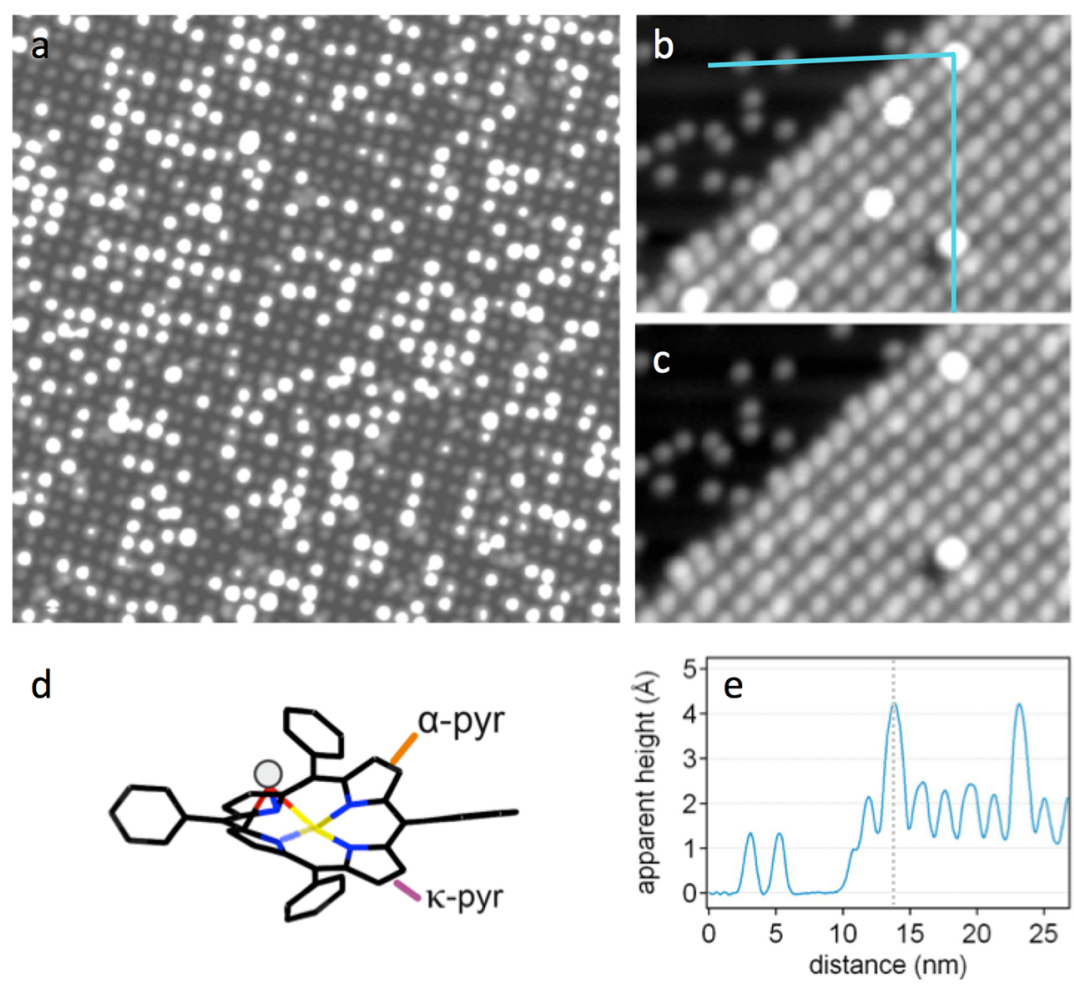

Figure 2. (a) Typical STM data after exposing the Co-TPP array (gray lattice) to an atomic beam of Fe. The Fe (white protrusions) follows the lattice of underlying Co-TPP $\left(I=30 \mathrm{pA}, V_{b}=-0.1 \mathrm{~V}, 443 \AA \times 443 \AA\right)$. (b) Zoom-in image representing a $\mathrm{Ag}(111)$ area partially covered by a Co-TPP islands. The blue line marks the height profile shown in (e) $\left(I=0.1 \mathrm{nA}, V_{\mathrm{b}}=-0.5 \mathrm{~V}\right.$, $219 \AA \times 150 \AA$ ). (c) Same area as shown in (b) after the deliberate removal of five Fe atoms from the Co-TPP template. (d) Model of a Co-TPP molecule in its adsorption geometry highlighting the saddle-shape distortion of the macrocycle and visualizing an Fe atom (gray ball) trapped on the porphyrin. (e) Height profile allowing for a direct comparison of the apparent height of Fe atoms on Co-TPP and Ag(111).

ground states of the $d^{7}$ and $d^{8}$ configurations assume low-spin configurations with $A_{1}$ symmetry, however, with dissimilar $S=1 / 2$ and $S=0$ for $d^{7}$ and $d^{8}$, respectively. Coupling the two $S=1 / 2$ of the substrate electron $E$ and the $d^{7}$ ground state generates a singlet $S$ $=0$ and a triplet $S=1$ state having the same energy. The hopping term mixes the $d^{7} E$ and $d^{8}$ configurations, which is effective between states having the same orbital symmetry and the same spin multiplicity. Hence, the singlet $S=0 d^{7} E$ state mixes with the $S=$ 0 state of the $d^{8}$ configuration, which results in a lowered energy of the mixed state. The nonbonding triplet $d^{7} E$ state remains in energy since it cannot mix with the lowest singlet $d^{8}$ state. Therefore, even very small mixing of the $d^{7} E$ and $d^{8}$ configurations, or in other words, fractional amount of electron transfer from the substrate to $\mathrm{Co}$, leads to spin quenching of the Co ground state. This quenching is not unique for the $\mathrm{C}_{4 \mathrm{~h}}$ symmetry. Further lowering the symmetry to $\mathrm{C}_{2 \mathrm{~h}}$, which occurs due to the saddle shape distortion of the molecules adsorbed on the surface, will modify slightly the energies of the ground state levels in the respective $d^{7}$ and $d^{8}$ configurations but will not further split the orbital $A_{1}$ singlets. Therefore, the same argument for spin quenching holds true also for lower symmetries.
This finding is consistent with the experimental data where no XMCD signal is present. Considering the parameters used in the multiplet calculation, the system is in a mixed-valence state. Comparing the XMCD data and the multiplet approach for the bare Co-TPP to our DFT calculations (cf. Computational Methods section), we find a very good overall agreement. DFT also predicts the Co-TPP adsorbed on Ag(111) to have no magnetic moment as found in the experiment. As visible from the orbital resolved density of states (DOS) shown in the Supporting Information (Figure $\mathrm{S} 1$ ) only the $\mathrm{d}_{z^{2}}$-orbital exhibits a considerable energy broadening as a result of strong hybridization upon adsorption, ${ }^{45}$ in agreement with the multiplet calculations including electron hopping only along the

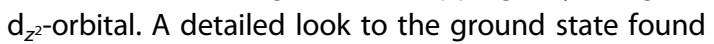
in the multiplet calculations reveals that the 2.75 holes in the Co d-orbitals are mainly localized on the $d_{x^{2}-y^{2}}$-orbital ( 0.024 electrons per spin channel) and the $d_{z^{2}}$-orbital (0.675 electrons per spin channel). These numbers agree qualitatively with the DFT calculations that find 2.34 holes in the Co d-orbitals. The DFT calculations, just as the multiplet calculations, determine the $d_{x^{2}-y^{2}}$ orbital to be the least occupied d-orbital. However, the DFT approach yields a higher occupation of this orbital (38\% of each spin orbital 
filled) as compared to the multiplet result. The difference in occupation is assigned to limitations of the multiplet approach, which neglects a macrocycle deformation and any orbital overlap between the $\mathrm{d}$-states and the ligand orbitals-in contrast to DFT. Also the $d_{z}{ }^{2}$-orbital is found to be the second least occupied of the d-orbitals (79\% of each spin orbital filled). The difference in the occupation of the outand in-plane oriented orbitals leads to the strong linear dichroism observed in the experiments (see Figure 1f).

Fe Decoration. The high structural regularity of the Co-TPP arrays on $\mathrm{Ag}(111)$ makes them promising templates for organizing adsorbate species. This potential was for example highlighted by the ligation of Co-TPP with small gas molecules as carbon monoxide or nitric monoxide. ${ }^{57,58}$ Here, the Co-TPP arrays were exposed in situ at $10 \mathrm{~K}$ to an atomic beam of Fe. A typical STM image after Fe deposition is shown in Figure 2a. Clearly, bright (white) protrusions in registry with the Co-TPP lattice (gray array) can be observed. At low coverage, most of these protrusions can be assigned to individual Fe atoms, while at higher coverage small Fe clusters are more prominent (vide infra).

A close-up STM image (Figure 2b) confirms that the bright protrusions appear near central positions of the Co-TPP molecules. These data were recorded on a $\mathrm{Ag}(111)$ surface not completely covered by Co-TPP, which allows us to quantify the Fe coverage (vide infra) and to directly compare the appearance of Fe atoms on $\mathrm{Ag}(111)$ and on Co-TPP. The line profile plotted in Figure 2e reveals an increased apparent height $(1.9 \AA$ at $-0.5 \mathrm{~V}$, with respect to the maximum height of (o-TPP) of $\mathrm{Fe}$ atoms positioned on the porphyrin compared to Fe/Ag(111) (1.3 ̊), evidencing a modified electronic environment for the Fe atom. Importantly, atomic manipulation experiments prove that the Fe decorates the Co-TPP core. Figure $2 c$ shows the area represented in Figure $1 \mathrm{~b}$ after controlled removal of five Fe atoms by voltage sweeps ( $V_{\mathrm{b}}$ from -1.6 to $1.6 \mathrm{~V}$ ) and reveals an intact subjacent Co-TPP array. Consequently, we conclude that the $\mathrm{Fe}$ is indeed adsorbed at the porphyrin core, ruling out a possible placement below or between Co-TPP molecules. Any attempts to laterally transfer $\mathrm{Fe}$ atoms between adjacent porphyrins failed, indicating a considerable site-specific interaction between the Fe and the macrocycle.

A careful inspection of high-resolution STM data reveals that the Fe atoms are not perfectly centered on the Co-TPP molecule, but occupy sites offered through a heptamer defined by the $\mathrm{Co}-\mathrm{N}-\mathrm{C}-\mathrm{C}-\mathrm{C}-\mathrm{N}$ cyclic subunit in a bisector plane slightly displaced from the center (cf. Figure 3a). In total, four equivalent positions are observed for Fe on Co-TPP, reflecting the symmetry of the saddle-shaped macrocycle ( $c f$. Figure $2 \mathrm{~d}$ ). The projected lateral distance of the Fe from the Co center
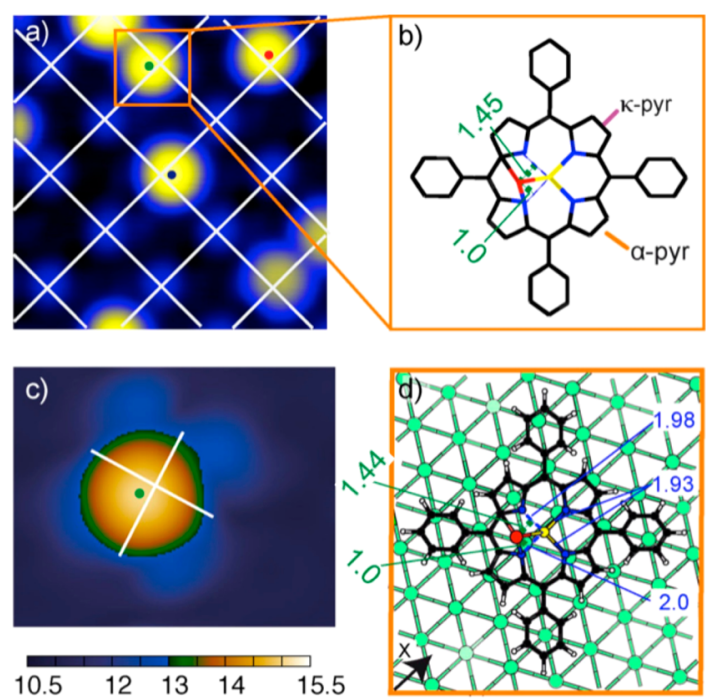

Figure 3. (a) High-resolution STM image revealing a minute off-center adsorption of the Fe atoms on the porphyrin lattice. $\left(I=0.1 \mathrm{nA}, V_{\mathrm{b}}=-0.1 \mathrm{~V}, 55.4 \AA \times 55.4 \AA\right)$. (b) Schematic model displaying the lateral Fe (marked in red) displacements from $\alpha$ and $\kappa$ directions extracted from the STM data. All numbers are in Å units. (c) Simulated constantcurrent STM image based on the structure shown in (d) $\left(V_{\mathrm{b}}=-0.2 \mathrm{~V}, 32 \AA \times 25 \AA\right)$. The given distances are in $\AA$ and relative to the bare $\mathrm{Ag}$ substrate, the overall corrugation amounts to $4.5 \AA$ A. (d) DFT optimized Fe/Co-TPP complex on $\mathrm{Ag}(111)$ in a $11 \times 5 \sqrt{ }(3)$ cell corroborating the off-center adsorption. The projected distances are indicated on the left and perfectly match the experimental values (compare (b)). The four $\mathrm{Co}-\mathrm{N}$ distances are quoted on the right to unveil the structural deformation upon Fe coordination. Green, black, blue, white, yellow and red balls depict respectively $\mathrm{Ag}, \mathrm{C}, \mathrm{N}, \mathrm{H}, \mathrm{Co}$ and Fe atoms. See text and Supporting Information for discussion.

extracted from STM data amounts to $1.8 \pm 0.2 \AA$, the symmetry reduction is evidenced by a larger separation of the projected Fe position from the $\alpha$-pyr axis $(1.45 \pm 0.2 \AA)$ as from the $\kappa$-pyr axis $(1.0 \pm 0.2 \AA)$ (cf. Figure 3b). A DFT optimization of the Fe/Co-TPP complex adsorbed on $\mathrm{Ag}(111)$ reveals that the Fe atom indeed binds preferentially in an off-center bisector position (cf. Figure 3d) with a binding energy of $1.87 \mathrm{eV}$. This configuration is energetically lower by $0.29 \mathrm{eV}$ as compared to a tilted on-top adsorption (described in Figure S3 in Supporting Information), thus corroborating the experimental observations. The Fe position on the adsorbed porphyrin macrocycle extracted from theory (projected distance Fe-Co $1.75 \AA$, Fe- $\alpha$-pyr $1.44 \AA$, Fe- $-\kappa$-pyr $1.00 \AA$ in Figure $3 \mathrm{~d}$ ) perfectly fits to the experimental values. The computed apparent height in STM simulations (cf. Figure $3 c$ ) and the measured scan profile of Figure 2e both agree on an overall corrugation of $\sim 4.5 \AA$, with respect to the bare Ag metal surface level.

Judging from the analysis of the most stable structure, Fe binds to three different atoms: one $\mathrm{N}$ from an $\kappa$-pyr group, one $C$ from an $\alpha$-pyr group, and the central $\mathrm{Co}$ atom. The $\mathrm{Fe}-\mathrm{C}$ bond length of $2.06 \AA$ corresponds to the values typically found in ferrocene 

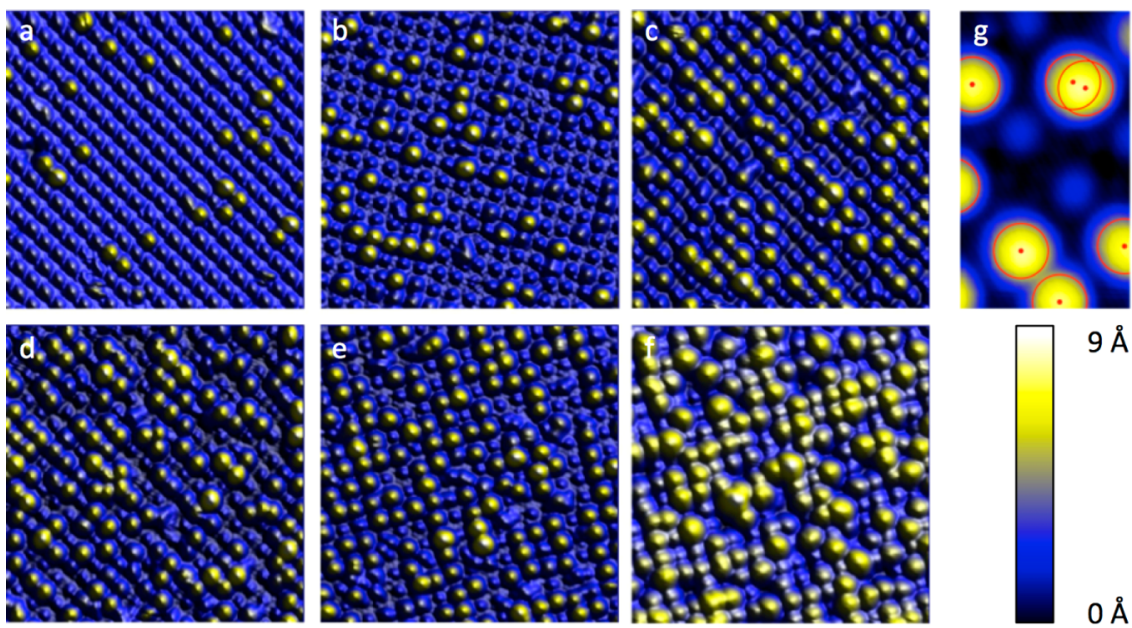

Figure 4. Series of STM images $(a-f)$ as a function of increasing Fe coverage $\Theta$ : Both the occupation $O$ of Co-TPP as well as the average cluster size $s$ increase. (Compare to simulated distributions in Figure S2 in the Supporting Information. The $s$ values are estimated from the simulations.) The color scale is adapted to highlight the Fe (yellow) on the Co-TPP array (blue). Throughout the whole coverage range, the Fe follows the square lattice of the porphyrin template (all image sizes $220 \AA \times$ $220 \AA ̊$ ). (a) $\Theta \approx 0.6 \% \mathrm{ML}, O \approx 13 \%, s \approx 1.2 ; l=0.16 \mathrm{nA}, V_{\mathrm{b}}=-0.5 \mathrm{~V}$., (b) $\Theta \approx 1.4 \% \mathrm{ML}, O \approx 25 \%, s \approx 1.5 ; I=79 \mathrm{pA}, V_{\mathrm{b}}=-0.1 \mathrm{~V}$. (c) $\Theta \approx 3 \% \mathrm{ML}, O \approx 51 \%, s \approx 1.6 ; I=67 \mathrm{pA}, V_{\mathrm{b}}=-0.5 \mathrm{~V}$. (d) $\Theta \approx 4.4 \% \mathrm{ML}, O \approx 65 \%, s \approx 1.8 ; I=0.1 \mathrm{nA}, V_{\mathrm{b}}=-0.1 \mathrm{~V}$. (e) $\Theta \approx 6 \% \mathrm{ML}$, $0 \approx 73 \%, s \approx 2 ; l=67 \mathrm{pA}, V_{\mathrm{b}}=-0.1 \mathrm{~V}$. (f) $\Theta \approx 10 \% \mathrm{ML}, O \approx 90 \%, s \approx 3 ; l=89 \mathrm{pA}, V_{\mathrm{b}}=-0.1 \mathrm{~V}$. (g) Zoom-in comparing the appearance of Fe/Co-TPP $(s=1)$ with a larger complex assigned to $\mathrm{Fe}_{2} / \mathrm{Co}-\mathrm{TPP}(s=2$, top right). The positions and the centerto-center distance of $3 \AA \AA$ between the overlaid red circles representing Fe atoms are consistent with bisector binding sites of both Fe units (cf. Figure $3 b, V_{b}=-0.1 \mathrm{~V}$ ).

$\left(\mathrm{FeCp}_{2}\right)$ species. ${ }^{59,60}$ At the same time, the $\mathrm{Fe}-\mathrm{Co}$ bond length $(2.45 \AA)$ is even slightly lower than the usual distances encountered in bimetallic or trimetallic metal complexes $(\sim 2.6 \AA)$, ${ }^{61,62}$ which indicates a strong metallic interaction (vide infra). This multiple hapticity of Fe permits to stabilize this highly reactive metal atom. The cobalt-porphyrin complex hence offers a very specific site for the Fe atom. Nevertheless, as evidenced by our calculations, the Fe adsorption does not induce a significant modification of the Co-TPP conformation (see Supporting Information, Figure S6). A further discussion addressing the impact of the Fe coordination on the porphyrin's electronic structure as well as the magnetic properties of the complex follows below.

Fe Coverage Dependence. Figure 4 summarizes the coverage dependent growth of Fe on the Co-TPP arrays. Already a simple visual inspection of the STM images reveals that the Fe follows the square lattice of the porphyrin template throughout the coverage range explored. As the Fe coverage is augmented, both the occupation of Co-TPP and the average cluster size increases. While individual Fe atoms dominate at low-coverage (Figure 4a), clusters reaching an apparent height of $9 \AA$ are observed at higher coverage (Figure 4f). Interestingly, even at the highest coverage of 0.1 monolayer, no coalescence of clusters is observed and no Fe is detected between porphyrins or at the molecular peripheries. Here, one monolayer (ML) is defined as one Fe atom per surface $\mathrm{Ag}$ atom. A full Co-TPP layer would thus correspond to a coverage of $0.037 \mathrm{ML}$. In contrast, an earlier study on room-temperature deposition of $\mathrm{Fe}$ on Fe-TPP
monolayers/Ag(111) reports the growth of compact two-dimensional Fe underneath the porphyrin array. ${ }^{63}$

Three values are used to describe the Fe morphology on the Co-TPP template layer: (i) the occupation $O$ of Co-TPP, where $0 \%$ corresponds to a bare Co-TPP array and $100 \%$ would correspond to a situation where every porphyrin is occupied by one or more Fe atoms. (ii) The total Fe coverage $\Theta$ in percentage of one ML. (iii) The average cluster size s, i.e., the average number of Fe atoms on an occupied porphyrin. These values are indicated in the caption of Figure 4 for each coverage. In order to precisely determine the Fe coverage, we used $\mathrm{Ag}(111)$ surfaces not completely covered by Co-TPP. This allows one to measure the density of individual Fe atoms on the bare Ag areas, from which a precise coverage calibration is derived. This procedure assumes that no Fe atoms are transferred between $\mathrm{Ag}$ and Co-TPP, an assumption fully justified at the low substrate temperature during deposition $(10 \mathrm{~K})$ where atomic diffusion and exchange processes are suppressed. As a result, we conclude that the majority of the protrusions at low coverage correspond to individual Fe atoms. However, already in this situation apparent height measurements reveal the existence of a small amount of dimers. A closer view of such a cluster is depicted in Figure $4 \mathrm{~g}$, suggesting that the monomer preferred binding site is retained.

At high coverage, the calibration procedure becomes less reliable, as Fe aggregation starts on the bare $\mathrm{Ag}$ areas and the classification of cluster sizes on Co-TPP by measuring the apparent cluster heights becomes difficult, which would result in considerable error bars for values $\Theta$ and s. To circumvent this 

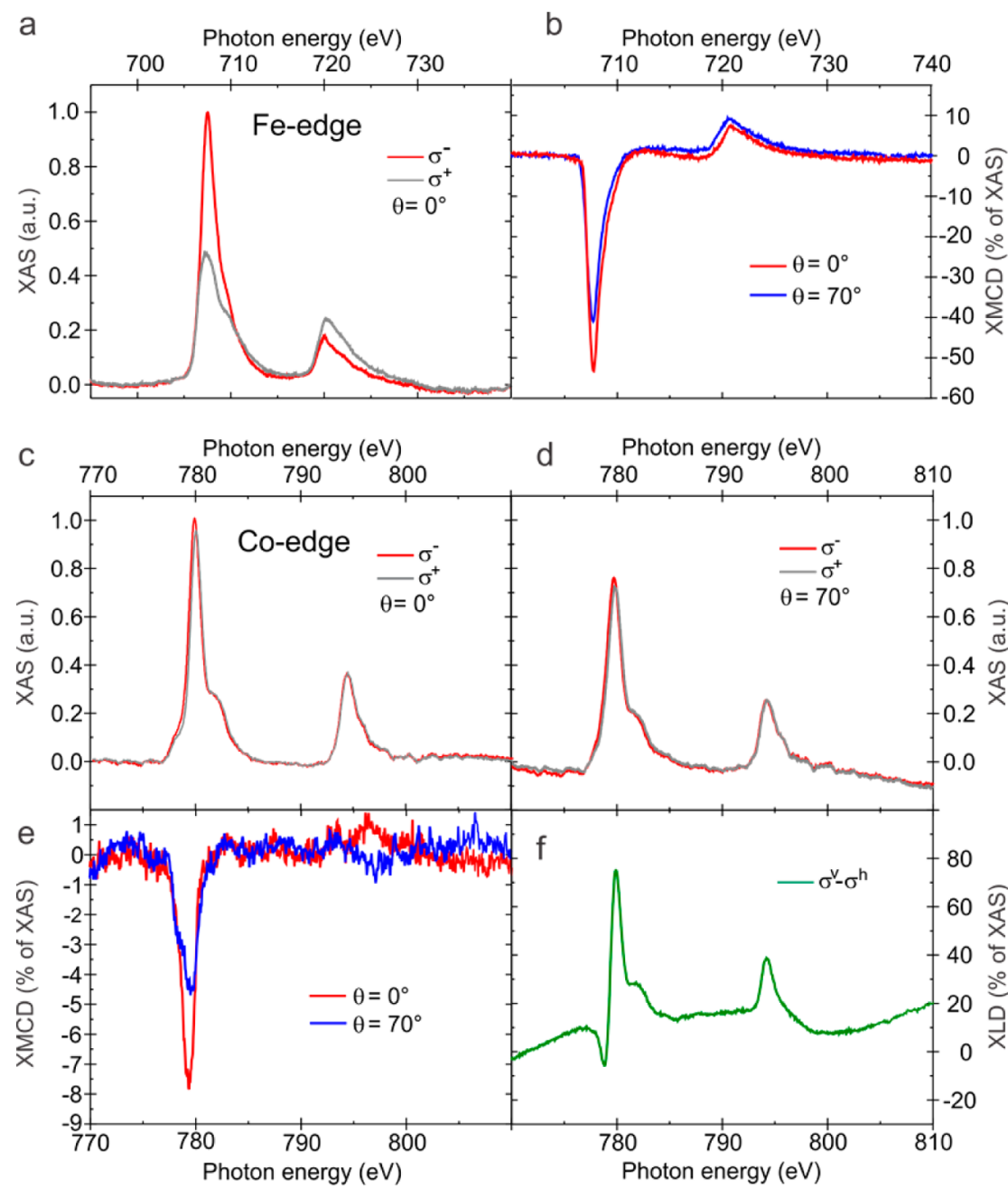

Figure 5. (a) XAS (at $\theta=0^{\circ}$ ) and (b) XMCD (both angles) for the Fe-edge. (c-f) XAS of the Co-edge. (c) XAS for $\theta=0^{\circ}$ and (d) $\theta=70^{\circ}$. (e) XMCD (both angles) for the Co-edge. (f) XLD measured at $\theta=70^{\circ}$. For all measurements $T=8 \mathrm{~K}$, (a-e) $B=5 \mathrm{~T}$, (f) $B=0.2 \mathrm{~T}$. For the XAS data in (a), (c), and (d), the Ag-background and a step function have been subtracted (cf. Supporting Information, Figure S4). All spectra were normalized to the maximum intensity of the corresponding Co- and $\mathrm{Fe}-\mathrm{L}_{3}$ edge, respectively. As for Figure 1 the Co XLD shows modulations resulting from the finite XLD of the Ag substrate.

problem, we compared our experimental data to a statistical simulation based on random adsorption in a hit-and-stick model (see Supporting Information, Figure S2). The fact that the modeled values for lowcoverage, where the experimental calibration is reliable, agree reasonably well with the experimental values leads us to conclude that the Fe morphology is mainly dictated by random adsorption with no indication for Fe transfer between porphyrins. This result is in agreement with the Fe confinement at the Co-TPP macrocycle discussed in the previous paragraph.

Magnetic Signature of Fe-Decorated Co-TPP. The XMCD measurements presented below were performed on a $\mathrm{Ag}(111)$ surface fully covered by Co-TPP to ensure that the Fe-signal originates exclusively from clusters adsorbed on the macrocycle (see Methods for details). For these measurements the Fe coverage was $\Theta=6 \pm 2 \%$. Therefore, the mean cluster size amounts to two Featoms and about $70 \%$ of the Co-centers are decorated with Fe. Figure 5 shows the XAS and XMCD data measured on the Co and Fe L-edge for the Fe decorated Co-TPP/Ag(111). The X-ray absorption spectra of the Fe edge (Figure 5a) show broad peaks expected for Fe clusters. The XMCD of the Fe (depicted in Figure $5 b$ ) is strong. On first glance the $\mathrm{X}$-ray absorption spectra measured on the Co-edge (Figure $5 c$,d) are only marginally influenced by the adsorption of Fe (cf. Figure 1c). The main difference is a shift of the shoulder in the $L_{3}$ edge toward the main peak. Surprisingly, the Co-edge now shows a finite XMCD (Figure 5e) in striking contrast to the bare Co-TPP array. This is a clear sign that the presence of Fe modifies the electronic/magnetic ground state of the $\mathrm{Co}$ and lifts the quenching mechanism discussed above.

As evidenced by the magnetization curves shown in Figure 6, we saturate the sample for the out-of-plane direction and can thus apply a sum-rule analysis to extract the average magnetic moments for $\mathrm{Fe}$ and Co. ${ }^{50,51}$ Since the extracted moments are proportional to the number of holes in the $d$-shell $\left(h_{\mathrm{d}}\right)$ of the specific 




Figure 6. Element resolved magnetization curves for Co and $\mathrm{Fe}$ along the out-of-plane and close to in-plane directions. The curves were obtained by measuring the XMCD signal at the corresponding $L_{3}$-edge as a function of magnetic field at $8 \mathrm{~K}$. The slight asymmetry in the Co magnetization curve is attributed to a measurement artifact induced by the low signal intensity.

atom, we use the values calculated by DFT for the Fe/Co-TPP complex, namely $h_{\mathrm{d}}=2.4$ for the Co center and $h_{\mathrm{d}}=3.3$ for one Fe atom. The experiment bases on an average of two Fe atoms per Co-TPP molecule. We assume that this does not change $h_{\mathrm{d}}$ of the Co center, but use $h_{\mathrm{d}}=3.65$ for an atom in the dimeric Fe cluster, assuming that 0.7 electrons are transferred to the $\mathrm{Fe}$ cluster irrespective if it consists of one, two or several atoms. Using these $h_{\mathrm{d}}$ values, we determine the effective spin moment per atom $\left(M_{S}+M_{7 D}\right)$ to be $2.2 \pm 0.3 \mu_{\mathrm{B}}\left(0.11 \pm 0.06 \mu_{\mathrm{B}}\right)$ and the orbital moment $\mathrm{M}_{\mathrm{L}}=0.51 \pm 0.07 \mu_{\mathrm{B}}\left(0.045 \pm 0.014 \mu_{\mathrm{B}}\right)$ for Fe (Co) (all values are for $\theta=0^{\circ}$ ). The large error bars are induced by the very small signals and a delicate subtraction of the large Ag background (see Supporting Information, Figure S4). The Co values consider that $\approx 30 \%$ of the Co signal is contributed from bare Co-TPP that does not possess any magnetic moment and consequently only refer to Co interacting with Fe (cf. Figure 4e). Clearly, the magnetic moments in $\mathrm{Co}$, induced by the presence of the $\mathrm{Fe}$, remain much smaller than expected for free spin 1/2 Co-TPP, giving evidence that the presence of the Fe cluster restores the magnetic moments only partially. It is also interesting to compare the Fe moments for the clusters to values of free Fe-clusters. Fe-ions forming isolated clusters having the size distribution of the ensembles in our study would have an effective spin moment of about $3.3 \mu_{\mathrm{B}}$ and an orbital moment of about $1 \mu_{\mathrm{B}} \cdot{ }^{64,65}$ As the spin dipole moment in the Fe-clusters on Co-TPP remains unknown, a direct comparison of the effective spin moment is difficult. However, a significantly reduced orbital moment is found for the $\mathrm{Fe}$ clusters adsorbed on the Co-TPP compared to free clusters, indicating the impact of the different coordination environment. The $\mathrm{Fe}-\mathrm{Fe}$ distance in free clusters is considerably shorter than the separation between Fe units in a $\mathrm{Fe}_{2} / \mathrm{Co}$-TPP complex suggested to bind in the bisector positions (compare Figure $4 \mathrm{~g}$ ).

The XLD of the Co-edge remains significant also after the adsorption of Fe. In fact, apart from the already discussed shift in the shoulder of the $\mathrm{L}_{3}$-edge there is little change, showing that the considerable imbalance in the occupation of the Co in-plane and out-of-plane orbitals remains.

The distinct dichroism in both Fe and Co allows us to trace the individual magnetization curves of the two elements. At our measuring conditions both curves almost reach saturation for $\theta=0^{\circ}$, but not for $70^{\circ}$ (Figure 6), implying an out-of plane easy axis. For the Fe case our ensemble measurement averages over several in-plane configurations and cluster sizes; i.e., we only report an effective out-of plane easy axis and do not claim that the easy axis of a Fe/Co-TPP complex is precisely perpendicular to the surface. As visible in Figure 6, the magnetization curves of Fe and Co have a similar shape. Considering that the Co moments are significantly smaller than the Fe moments the similar shape is a clear demonstration of appreciable ferromagnetic coupling that is sufficient to compete with thermal fluctuations of $k_{\mathrm{B}} T \approx 0.7 \mathrm{meV}$.

Theoretical Description of the $\mathrm{Fe} / \mathrm{Co}_{0} \mathrm{TPP}$ Complex. Finally we analyze the magnetic properties by means of DFT and address the magnetic interaction between Fe and its nearest neighbors. The spin-polarized calculations conducted on the Fe/Co-TPP complex adsorbed on $\mathrm{Ag}(111)$ reveal a significant magnetization of $\approx 2.9$ unpaired electrons, while in the absence of Fe no magnetization is seen for Co-TPP/Ag(111), supporting the experimental results. The spin density map representing the difference between spin-up and spindown electron densities displayed in Figure 7a unveils three important aspects. First, this magnetization is mostly localized on the Fe (2.74 unpaired electrons, see Table S1 in Supporting Information), while a small component is induced on the Co metal center (0.18 unpaired electrons). No significant magnetization is seen on the $\mathrm{C}$ or $\mathrm{N}$ atoms of the TPP macrocycle; i.e., the tetradentate ligand only plays an indirect role in the magnetic coupling. Second, the magnetization has the same sign on both the Fe and Co atoms. Third, the shape of the contour enables one to identify the nature of the $\mathrm{d}$-orbitals participating in the magnetization.

Concerning $\mathrm{Fe}$, the dip along $z$ (normal axis) is coherent with $d_{x z}$ and $d_{y z}$ states and the alternating dips/protrusions in the $x y$ plane (i.e., in the porphyrin plane) clearly indicate the contribution of $d_{x^{2}-y^{2}}$ states mixing with $d_{x y}$ orbitals. Concerning $\mathrm{Co}$, we learn from the calculated spin-density maps that the compact shape arises from a mixture of $d_{x z}$ and $d_{y z}$ states. These conclusions are fully confirmed by the detailed analysis of the DOS projected onto the $d$-shell of the magnetic atoms (see Supporting Information Figure S5). We rationalize this observation by an orbital scheme where the total $d$ occupation amounts to 7.6 electrons on Co $\left(h_{d}=2.4\right.$, vide supra) and 6.7 electrons on Fe $\left(h_{d}=3.3\right)$, respectively (see Figure $7 c$ ). These numbers are based on a quantitative evaluation of the occupation of the 

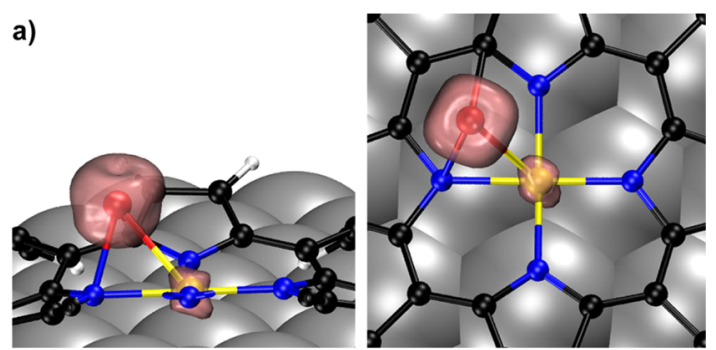

b)

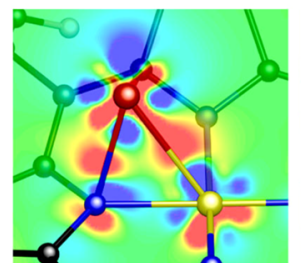

c)
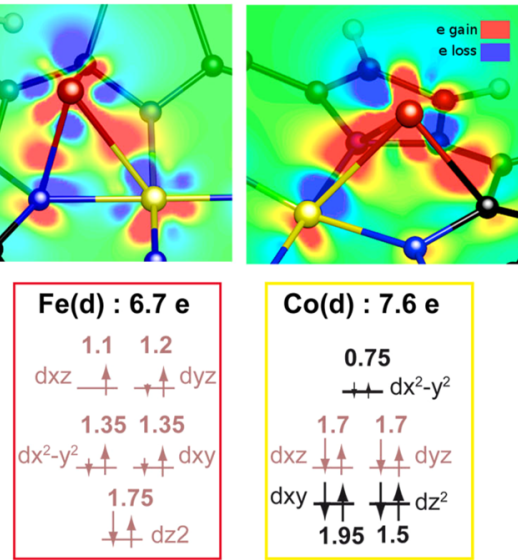

Co(d) : $7.6 \mathrm{e}$

0.75

$+4 \mathrm{dx}^{2}-\mathrm{y}^{2}$

$1.7 \quad 1.7$

$d x z+4+4 d y z$

$\mathrm{dxy} \downarrow 4+4 \mathrm{dz}^{2}$

1.951 .5

Figure 7. (a) Side and top view of the calculated spin density map (difference between spin-up and spin-down electron densities). The total magnetization of 2.9 unpaired electrons is majorly localized on the $\mathrm{Fe}$ atom (prominent mixture of $d_{x z \prime}$ $d_{y z}$ and minor contribution of $d_{x^{2}-y^{2}}$ ) and minorly on the Co center (mixture of $d_{x z}$ and $d_{y z}$ states). The magnetization on both atoms has the same sign colored in antique pink. (b) Charge redistribution upon Fe ligation displayed on two binding planes: $\mathrm{Fe}-\mathrm{Co}-\mathrm{N}$ (left panel) and $\mathrm{Fe}-\mathrm{Co}-\mathrm{C}$ plane (right panel). The atoms behind the respective planes are shaded in green. The electron gain areas (red) mostly localize in between atoms and also in the d-shell of Fe. The electron loss zones (blue) are mainly localized on the magnetic metals. (c) Level scheme of the PDOS projected onto the d-manifold of the $\mathrm{Fe}$ atom and the $\mathrm{Co}$ center in the adsorbed $\mathrm{Fe} / \mathrm{Co}$-TPP complex (see Supporting Information, Figure S3). The magnetic (nonmagnetic) $d$-shells are colored in antique pink (black), respectively. The occupation numbers derive from Table S1 in the Supporting Information. The z-axis is the normal direction to the metallic surface.

d-manifold of $\mathrm{Co}$ and $\mathrm{Fe}$ in both spin channels (see Supporting Information Table S1). In addition, maps representing the charge difference upon Fe adsorption shown in Figure $7 \mathrm{~b}$ reveal a complex electron redistribution induced by the presence of Fe. Clearly electrons are shared in between bonding atoms (see the red zones representing increased electronic population) indicating covalent interactions. This evidences a strong bonding of Fe encapsulated by the reactive site formed by the Co center and the closest $\mathrm{N}$ and $\mathrm{C}$ atoms, consistent with the short bond distances reported above. At the same time, a redistribution of the electron density in the $\mathrm{d}$-shells of the magnetic atoms occurs (see the red and blue zones with classical $d$ orbital shapes). Hence the Fe atom is shown to maximize its coordination to the supporting atoms by positioning at the center of the largest available ring, the cyclic heptameric $\mathrm{Co}-\mathrm{N}-\mathrm{C}-\mathrm{C}-\mathrm{C}-\mathrm{N}$ subunit, at the interface. This behavior is at odds with classical gaseous ligands such as NO or CO being single adducts $^{38,49}$ or bridging metal-pyrrole bonds, ${ }^{57}$ respectively and complements unusual geometries reported for tetrapyrroles. ${ }^{42-44}$ The shared covalency of Fe with nonmagnetic $\mathrm{N}$ and $\mathrm{C}$ atoms furthermore may imply a dual mechanism to account for the ferromagnetism: a direct coupling and an indirect superexchange contribution mediated by the covalently bond $\mathrm{N}$ and $\mathrm{C}$ atoms.

Comparing the DFT results to the XMCD measurements we find a very good qualitative agreement, despite limitations induced by the unknown dipolar term in the XMCD analysis. First, both demonstrate that the Co-center once in contact to Fe regains a finite magnetic moment, which however remains much smaller than the Fe moments. Second, experiment and theory both yield a ferromagnetic coupling between the two metals. Also the strong imbalance in the orbital occupation for in-plane and out-of-plane orbitals, evidenced in the strong XLD of Co, is clearly seen in DFT for both Fe covered and bare Co-TPP.

\section{CONCLUSIONS}

We demonstrated the site-specific binding of $\mathrm{Fe}$ atoms and small clusters on highly regular Co-porphyrin templates on $\mathrm{Ag}(111)$. The special coordination environment offered by the Co center, one $\mathrm{N}$ and one $\mathrm{C}$ atom and the way a single Fe atom is attached therein have been identified unequivocally. This bonding configuration, characterized by a calculated $\mathrm{Fe}-\mathrm{Co}$ distance of $2.45 \AA$, yields four distinct and equivalent $\mathrm{Fe}$ positions on the Co-TPP, which are all identified in the STM experiments. Thus, our results provide access to heterometal bonding at metalloporphyrins, extending the understanding of tetrapyrrole units exhibiting unusual geometries and providing hitherto unrecognized pathways for surface coordination systems and molecular spintronics. By increasing the Fe coverage, the average Fe clusters size can be controlled, while site-specific confinement of $\mathrm{Fe}$ at the porphyrin macrocycle persists. Consequently, using Co-TPP/Ag(111) as template, ultrahigh density arrays of Fe clusters separated from the metallic substrate can be fabricated thereby hindering the coalesence up to appreciable Fe coverages. Our XMCD measurements, corroborated by a multiplet analysis and DFT calculations, reveal that the Fe attachment revives the magnetic moment of the Co center, which is quenched upon adsorption on the $\mathrm{Ag}(111)$ surface. We report a ferromagnetic coupling between $\mathrm{Fe}$ and $\mathrm{Co}$, with an out-of-plane easy axis, where the nonmagnetic $\mathrm{N}$ and $\mathrm{C}$ atoms engage in a shared covalency with $\mathrm{Fe}$, suggesting a dual (direct and indirect) interaction mechanism. To the best of our knowledge this is the first study that shows the effect of magnetic species adsorbed on a metal-organic surface layer not only to steer the magnetic properties of the cluster, but also to induce an otherwise 
quenched moment in the molecular film. Consequently, it opens up pathways to engineer magnetic moments in well-defined nanoarchitectures supported on surfaces that are addressable with atomic precision and are based on novel bonding motifs for transition metal atoms on molecular templates.

\section{METHODS}

Experimental Section. The scanning tunneling microscopy (STM) experiments were performed in a custom-designed ultrahigh vacuum (UHV) apparatus comprising a commercial low-temperature STM (CreaTec, www.lt-stm.com). The system's base pressure is below $2 \times 10^{-10}$ mbar. The $\mathrm{Ag}(111)$ single crystal surface was cleaned by repeated cycles of $\mathrm{Ar}^{+}$sputtering (800 eV) followed by annealing to $730 \mathrm{~K}$. Subsequently, Co-TPP was deposited by organic molecular beam epitaxy (OMBE) from a quartz crucible held at $625 \mathrm{~K}$. Co-TPP was thoroughly degassed prior to any experiments resulting in a background pressure in the $10^{-10}$ mbar range during deposition. In our experiments we used Co-TPP acquired from a commercial supplier (Porphyrin Systems) at $98 \%$ purity. After dosing CoTPP at room temperature, the sample was cooled down and transferred into the STM chamber, where the Co-TPP array quality was checked by constant current images recorded at about $10 \mathrm{~K}$ using electrochemically etched tungsten tips. In the figure captions $V_{\mathrm{b}}$ refers to the bias voltage applied to the sample and / to the tunneling current. All STM images were processed using the WsXM software. ${ }^{66}$ Subsequently, Fe atoms were deposited in situ, directly onto the cold sample, by thermal evaporation from a home-built water-cooled cell. Throughout the experiments, the evaporator was run at constant power while the Fe dose was augmented by increasing the deposition time.

The XMCD measurements were performed at the ID08 beamline at the European Synchrotron facility in Grenoble in the total electron yield mode. The $\mathrm{Ag}(111)$ crystals have been cleaned using the above descripted procedure of Ar-sputtering and annealing to $730 \mathrm{~K}$. For the Co-TPP, a multilayer of the metal organic complexes have been directly deposited on the sample at $300 \mathrm{~K}$. Annealing the sample to $525 \mathrm{~K}$ leads to desorption of the multilayer leaving one complete monolayer on the sample surface. It is important to note that this procedure ensures a full coverage of the $\mathrm{Ag}(111)$ surface by one monolayer of a highly ordered intact Co-TPP film. This is helpful to maximize the Co-signal in the measurements and more importantly it guarantees that the Fe-signal measured after its deposition results exclusively from Fe on top of the Co-TPP array. Before the sample was transferred in situ into the X-ray chamber, prior to $\mathrm{Fe}$ deposition, the sample quality was checked in situ with an STM at room temperature. The X-ray absorption measurements were performed at $\mathrm{T}=8 \mathrm{~K}$ with the measuring geometry sketched in the inset of Figure 1c. First for every sample the X-ray absorption of the bare Co-TPP was measured and then Fe was deposited in situ with the sample kept at $8 \mathrm{~K}$. The Fe coverage was determined in two steps. After calibrating the XAS signal with STM data on Fe clusters on $\mathrm{Ag}(111)$ and Fe in a supramolecular network on $\mathrm{Ag}(111)$, we used the XAS signal as a measure for the coverage. For the magnetization curves we measured the dichroism $\left(\sigma^{+}-\sigma^{-}\right)$at the maximum of the $\mathrm{L}_{3}$-edge normalized to the pre-edge absorption intensity as a function of applied magnetic field.

Computational Methods (DFT+STM, multiplet). The calculations were performed using density functional theory as implemented in the Vienna $A b$ Initio Simulation Package. ${ }^{67}$ This so-called VASP program uses a plane-wave basis set and the projectoraugmented plane wave method to account for electron-ion interactions. The generalized-gradient approximation exchangecorrelation functional parametrized by Perdew et al. ${ }^{68}$ was employed and in order to describe long-range van der Waals interactions, we used the semi empirical dispersion correction by Grimme ${ }^{69}$ as implemented in VASP. The calculations were performed with spin polarization and a $300 \mathrm{eV}$ energy cutoff. We considered a periodic slab of a $\mathrm{Ag}(111)$ surface with a size of $11 \cdot 5 \sqrt{3}$ unit cells in lateral dimensions and a vacuum region of $19.3 \AA$, which corresponds to approximately 6.5 unit cells. Only the top layer was relaxed to allow for interactions with the adsorbed molecule on one side of the slab. Because of the lateral size of the periodic cell, the k-point sampling was restricted to the $\Gamma$-point. The adsorption site for the Co-TPP molecule was determined prior to Fe deposition to be a bridge site $(\mathrm{BE}(\mathrm{Co}-\mathrm{TPP})=5.09 \mathrm{eV})$ with the $\kappa$-pyr axis aligning with the closed-packed directions of the $A g(111)$ surface lattice. Several initial positions of an Fe atom on adsorbed Co-TPP were explored: atop the central Co atom and off-center along the bisector line of two $\mathrm{Co}-\mathrm{N}$ directions. All these intitial adsorption structures yield the two presented optimized structures but a more complete survey of metastable positions of Fe will be the subject of a forthcoming paper. Finally constant current STM images at $-0.2 \mathrm{~V}$ were calculated using Tersoff-Hamann theory ${ }^{70}$ with a specific treatment of the wave function decay in the vacuum region. ${ }^{71}$

Multiplet Calculations. The crystal field parameters $\left(10 D_{\mathrm{q}}=\right.$ 2.5, $D_{\mathrm{s}}=0.38, D_{\mathrm{t}}=0.18 \mathrm{eV}$ ) are the same for the initial and final states. The Slater-Condon parameters were scaled to 0.68 of their atomic Hartree-Fock values. Configuration interaction between $d^{7} E$ and $d^{8}$ was allowed through the $d_{z^{2}}$ orbital with a hopping parameter of $t=1 \mathrm{eV}$. The charge transfer energy defined as the difference between the center of gravity of the $d^{8}$ and $d^{7} E$ states amounts to $1 \mathrm{eV}$ and $0 \mathrm{eV}$ for the initial and final state configurations. The finite temperature was taken into account by considering the Boltzmann distribution for the lowest lying energy states.

Conflict of Interest: The authors declare no competing financial interest.

Acknowledgment. Work supported by the ERC Advanced Grant MolArt (No. 247299), the German Research Foundation (DFG) through BA 3395/2-1, the Munich Center for Advanced Photonics (MAP) and the Technische Universität München Institute for Advanced Study, funded by the German Excellence Initiative. M.L.B, W.A. and J.V.B. thank the collaborative transnational grant in the ANR-DFG 2011 call (ANR-11-INTB-1014-01) for support. U.S. acknowledges funding by the Emmy-Noether program of Deutsche Forschungsgemeinschaft.

Supporting Information Available: Additional DFT calculations including PDOS plots, occupation numbers and adsorption configurations, raw XAS data. Simulated particle distribution. This material is available free of charge via the Internet at http://pubs.acs.org.

\section{REFERENCES AND NOTES}

1. Hirjibehedin, C. F.; Lin, C.-Y.; Otte, A. F.; Ternes, M.; Lutz, C. P.; Jones, B. A.; Heinrich, A. J. Large Magnetic Anisotropy of a Single Atomic Spin Embedded in a Surface Molecular Network. Science 2007, 317, 1199-1203.

2. Komeda, T.; Isshiki, H.; Liu, J.; Zhang, Y.; Lorente, N.; Katoh, K.; Breedlove, B. K.; M, Y. Observation and Electric Current Control of a Local Spin in a Single-Molecule Magnet. Nat. Commun. 2011, 2, 217.

3. Khajetoorians, A. A.; Baxevanis, B.; Hübner, C.; Schlenk, T.; Krause, S.; Wehling, T. O.; Lounis, S.; Lichtenstein, A.; Pfannkuche, D.; Wiebe, J.; et al. Current-Driven Spin Dynamics of Artificially Constructed Quantum Magnets. Science 2013, 339, 55-59.

4. Leuenberger, M. N.; Loss, D. Quantum Computing in Molecular Magnets. Nature 2001, 410, 789-793.

5. Bogani, L.; Wernsdorfer, W. Molecular Spintronics Using Single-Molecule Magnets. Nat. Mater. 2008, 7, 179-186.

6. Vincent, R.; Klyatskaya, S.; Ruben, M.; Wernsdorfer, W.; Balestro, F. Electronic Read-out of a Single Nuclear 
Spin Using a Molecular Spin Transistor. Nature 2012, 488, 357-360.

7. Miyamachi, T.; Schuh, T.; Märkl, T.; Bresch, C.; Balashov, T.; Stöhr, A.; Karlewski, C.; André, S.; Marthaler, M.; Hoffmann, M.; et al. Stabilizing the Magnetic Moment of Single Holmium Atoms by Symmetry. Nature 2013, 503, 242-246.

8. Barth, J. V.; Costantini, G.; Kern, K. Engineering Atomic and Molecular Nanostructures on Surfaces. Nature 2005, 437, 671-679.

9. Vitali, L.; Fabris, S.; Conte, A. M.; Brink, S.; Ruben, M.; Baroni, S.; Kern, K. Electronic Structure of Surface-Supported Bis(Phthalocyaninato)Terbium(III) Single Molecular Magnets. Nano Lett. 2008, 8, 3364-3368.

10. Stepanow, S.; Honolka, J.; Gambardella, P.; Vitali, L.; Abdurakhmanova, N.; Tseng, T.-C.; Rauschenbach, S.; Tait, S. L.; Sessi, V.; Klyatskaya, S.; et al. Spin and Orbital Magnetic Moment Anisotropies of Monodispersed Bis(Phthalocyaninato)Terbium on a Copper Surface. J. Am. Chem. Soc. 2010, 132, 11900-11901.

11. Umbach, T. R.; Bernien, M.; Hermanns, C. F.; Krüger, A.; Sessi, V.; Fernandez-Torrente, I.; Stoll, P.; Pascual, J. I.; Franke, K. J.; Kuch, W. Ferromagnetic Coupling of Mononuclear Fe Centers in a Self-Assembled Metal-Organic Network on Au(111). Phys. Rev. Lett. 2012, 109, 267207.

12. Abdurakhmanova, N.; Tseng, T.-C.; Langner, A.; Kley, C. S.; Sessi, V.; Stepanow, S.; Kern, K. Superexchange-Mediated Ferromagnetic Coupling in Two-Dimensional Ni-TCNQ Networks on Metal Surfaces. Phys. Rev. Lett. 2013, 110, 027202.

13. Wäckerlin, C.; Nowakowski, J.; Liu, S.-X.; Jaggi, M.; Siewert, D.; Girovsky, J.; Shchyrba, A.; Hählen, T.; Kleibert, A.; Oppeneer, P. M.; et al. Two-Dimensional Supramolecular Electron Spin Arrays. Adv. Mater. 2013, 25, 2404-2408.

14. Wegner, D.; Yamachika, R.; Zhang, X.; Wang, Y.; Baruah, T.; Pederson, M. R.; Bartlett, B. M.; Long, J. R.; Crommie, M. F. Tuning Molecule-Mediated Spin Coupling in Bottomup-Fabricated Vanadium-Tetracyanoethylene Nanostructures. Phys. Rev. Lett. 2009, 103, 087205.

15. Loth, S.; Baumann, S.; Lutz, C. P.; Eigler, D. M.; Heinrich, A. J. Bistability in Atomic-Scale Antiferromagnets. Science 2012, 335, 196-199.

16. Auwärter, W.; Ecija, D.; Klappenberger, F.; Barth, J. V. Porphyrins at Interfaces. Nat. Chem. 2015, 7, 105-120.

17. Scheybal, A.; Ramsvik, T.; Bertschinger, R.; Putero, M.; Nolting, F.; Jung, T. A. Induced Magnetic Ordering in a Molecular Monolayer. Chem. Phys. Lett. 2005, 4, 214-220.

18. Wende, H.; Bernien, M.; Luo, J.; Sorg, C.; Ponpandian, N.; Kurde, J.; Miguel, J.; Piantek, M.; Xu, X.; Eckhold, P.; et al. Substrate-Induced Magnetic Ordering and Switching of Iron Porphyrin Molecules. Nat. Mater. 2007, 6, 516-520.

19. Schwöbel, J.; Fu, Y.; Brede, J.; Dilullo, A.; Hoffmann, G.; Klyatskaya, S.; Ruben, M.; Wiesendanger, R. Real-Space Observation of Spin-Split Molecular Orbitals of Adsorbed Single-Molecule Magnets. Nat. Commun. 2012, 3, 953.

20. Lazarovits, B.; Szunyogh, L.; Weinberger, P. Fully Relativistic Calculation of Magnetic Properties of $\mathrm{Fe}, \mathrm{Co}$, and $\mathrm{Ni}$ Adclusters on Ag(100). Phys. Rev. B: Condens. Matter Mater. Phys. 2002, 65, 104441.

21. Tsukahara, N.; Noto, K.-i.; Ohara, M.; Shiraki, S.; Takagi, N.; Takata, Y.; Miyawaki, J.; Taguchi, M.; Chainani, A.; Shin, S.; et al. Adsorption-Induced Switching of Magnetic Anisotropy in a Single Iron(II) Phthalocyanine Molecule on an Oxidized Cu(110) Surface. Phys. Rev. Lett. 2009, 102, 167203.

22. Brede, J.; Atodiresei, N.; Kuck, S.; Lazic, P.; Caciuc, V.; Morikawa, Y.; Hoffmann, G.; Blügel, S.; Wiesendanger, R. Spin- and Energy-Dependent Tunneling through a Single Molecule with Intramolecular Spatial Resolution. Phys. Rev. Lett. 2010, 105, 047204.

23. Fanetti, M.; Calzolari, A.; Vilmercati, P.; Castellarin-Cudia, C.; Borghetti, P.; Santo, G. D.; Floreano, L.; Verdini, A.; Cossaro, A.; Vobornik, I.; et al. Structure and Molecule Substrate Interaction in a Co-Octaethyl Porphyrin Monolayer on the Ag(110) Surface. J. Phys. Chem. C 2011, 115, 1156011568.

24. Hu, Z.; Li, B.; Zhao, A.; Yang, J.; Hou, J. G. Electronic and Magnetic Properties of Metal Phthalocyanines on Au(111)
Surface: A First-Principles Study. J. Phys. Chem. C 2008, 112, 13650-13655.

25. Krenner, W.; Klappenberger, F.; Kühne, D.; Diller, K.; Qu, Z.-R.; Ruben, M.; Barth, J. V. Positioning of Single Co Atoms Steered by a Self-Assembled Organic Molecular Template. J. Phys. Chem. Lett. 2011, 2, 1639-1645.

26. Decker, R.; Schlickum, U.; Klappenberger, F.; Zoppellaro, G.; Klyatskaya, S.; Ruben, M.; Barth, J. V.; Brune, H. Using MetalOrganic Templates to Steer the Growth of Fe and Co Nanoclusters. Appl. Phys. Lett. 2008, 93, 243102-243102.

27. Makarova, M.; Mandal, S. K.; Okawa, Y.; Aono, M. Ordered Monomolecular Layers as a Template for the Regular Arrangement of Gold Nanoparticles. Langmuir 2013, 29, 7334-7343.

28. Vijayaraghavan, S.; Ecija, D.; Auwärter, W.; Joshi, S.; Seufert, K.; Seitsonen, A. P.; Tashiro, K.; Barth, J. V. Selective Supramolecular Fullerene-Porphyrin Interactions and Switching in Surface-Confined $\mathrm{C} 60-\mathrm{Ce}(\mathrm{TPP})_{2}$ Dyads. Nano Lett. 2012, 12, 4077-4083.

29. Robles, R.; Lorente, N.; Isshiki, H.; Liu, J.; Katoh, K.; Breedlove, B. K.; Yamashita, M.; Komeda, T. Spin Doping of Individual Molecules by Using Single-Atom Manipulation. Nano Lett. 2012, 12, 3609-3612.

30. Krull, C.; Robles, R.; Mugarza, A.; Gambardella, P. Site- and Orbital-Dependent Charge Donation and Spin Manipulation in Electron-Doped Metal Phthalocyanines. Nat. Mater. 2013, 12, 337-343.

31. Stepanow, S.; Rizzini, A. L.; Krull, C.; Kavich, J.; Cezar, J. C.; Yakhou-Harris, F.; Sheverdyaeva, P. M.; Moras, P.; Carbone, C.; Ceballos, G.; et al. Spin Tuning of Electron-Doped Metal-Phthalocyanine Layers. J. Am. Chem. Soc. 2014, 136, 5451-5459.

32. Miguel, J.; Hermanns, C. F.; Bernien, M.; Krüger, A.; Kuch, W. Reversible Manipulation of the Magnetic Coupling of Single Molecular Spins in Fe-Porphyrins to a Ferromagnetic Substrate. J. Phys. Chem. Lett. 2011, 2, 1455-1459.

33. Wäckerlin, C.; Chylarecka, D.; Kleibert, A.; Müller, K.; lacovita, C.; Nolting, F.; Jung, T. A.; Ballav, N. Controlling Spins in Adsorbed Molecules by a Chemical Switch. Nat. Commun. 2010, 1, 61.

34. Kim, H.; Chang, Y. H.; Lee, S.-H.; Kim, Y.-H.; Kahng, S.-J. Switching and Sensing Spin States of Co-Porphyrin in Bimolecular Reactions on Au(111) Using Scanning Tunneling Microscopy. ACS Nano 2013, 7, 9312-9317.

35. Wäckerlin, C.; Tarafder, K.; Girovsky, J.; Nowakowski, J.; Hählen, T.; Shchyrba, A.; Siewert, D.; Kleibert, A.; Nolting, F.; Oppeneer, P. M.; et al. Ammonia Coordination Introducing a Magnetic Moment in an on-Surface Low-Spin Porphyrin. Angew. Chem., Int. Ed. 2013, 52, 1-5.

36. Gambardella, P.; Stepanow, S.; Dmitriev, A.; Honolka, J.; de Groot, F. M. F.; Lingenfelder, M.; Gupta, S. S.; Sarma, D. D.; Bencok, P.; Stanescu, S.; et al. Supramolecular Control of the Magnetic Anisotropy in Two-Dimensional HighSpin Fe Arrays at a Metal Interface. Nat. Mater. 2009, 8, 189-193.

37. Hieringer, W.; Flechtner, K.; Kretschmann, A.; Seufert, K.; Auwärter, W.; Barth, J. V.; Görling, A.; Steinrück, H.-P.; Gottfried, J. M. The Surface Trans Effect: Influence of Axial Ligands on the Surface Chemical Bonds of Adsorbed Metalloporphyrins. J. Am. Chem. Soc. 2011, 133, 6206-6222.

38. Seufert, K.; Auwärter, W.; Barth, J. V. Discriminative Response of Surface-Confined Metalloporphyrin Molecules to Carbon and Nitrogen Monoxide. J. Am. Chem. Soc. 2010, 132, 18141-18146.

39. Barth, J. V. Fresh Perspectives for Surface Coordination Chemistry. Surf. Sci. 2009, 603, 1533-1541.

40. Daphnomili, D.; Scheidt, W. R.; Zajicek, J.; Coutsolelos, A. G. Heterometallic Homo- and Heteroleptic Porphyrinate Dimers with a Rhodium-Thallium Bond. Inorg. Chem. 1998, 37, 3675-3681.

41. Collman, J. P.; Boulatov, R.; Jameson, G. B. The First Quadruple Bond between Elements of Different Groups. Angew. Chem., Int. Ed. 2001, 40, 1271-1274.

42. Callot, H. J.; Tschnnibrr, T.; Chewier, B.; Weiss, R. Insertion of an Ethoxycarbonylmethylene Moiety into a Ni-N Bond of 
meso-Tetraphenylporphinatonickel(II). Angew. Chem., Int. Ed. 1975, 14, 567-568.

43. Latos-Grazynski, L.; Cheng, R.-J.; Mar, G. N. L.; Balch, A. L. Reversible Migration of an Axial Carbene Ligand into an Iron-Nitrogen Bond of a Porphyrin. Implications for High Oxidation States of Heme Enzymes and Heme Catabolism. J. Am. Chem. Soc. 1981, 103, 4270-4272.

44. Tatsumi, K.; Hoffmann, R. Metalloporphyrins with Unusual Geometries. 2. Slipped and Skewed Bimetallic Structures, Carbene and Oxo Complexes, and Insertions into MetalPorphyrin Bonds. Inorg. Chem. 1981, 20, 3771-3784.

45. Auwärter, W.; Seufert, K.; Klappenberger, F.; Reichert, J.; Weber-Bargioni, A.; Verdini, A.; Cvetko, D.; Dell'Angela, M.; Floreano, L.; Cossaro, A.; et al. Site-Specific Electronic and Geometric Interface Structure of Co-Tetraphenyl-Porphyrin Layers on Ag(111). Phys. Rev. B: Condens. Matter Mater. Phys. 2010, 81, 245403.

46. Comanici, K.; Buchner, F.; Flechtner, K.; Lukasczyk, T.; Gottfried, J. M.; Steinrück, H.-P.; Marbach, H. Understanding the Contrast Mechanism in Scanning Tunneling Microscopy (STM) Images of an Intermixed Tetraphenylporphyrin Layer on Ag(111). Langmuir 2008, 24, 1897-1901.

47. Lukasczyk, T.; Flechtner, K.; Merte, L. R.; Jux, N.; Maier, F.; Gottfried, J. M.; Steinrück, H.-P. Interaction of Cobalt(II) Tetraarylporphyrins with a $\mathrm{Ag}(111)$ Surface Studied with Photoelectron Spectroscopy. J. Phys. Chem. C 2007, 111, 3090-3098.

48. Buchner, F.; Warnick, K.-G.; Wölfle, T.; Görling, A.; Steinrück, H.-P.; Hieringer, W.; Marbach, H. Chemical Fingerprints of Large Organic Molecules in Scanning Tunneling Microscopy: Imaging Adsorbate-Substrate Coupling of Metalloporphyrins. J. Phys. Chem. C 2009, 113, 16450-16457.

49. Burema, S. R.; Seufert, K.; Auwärter, W.; Barth, J. V.; Bocquet, M.-L. Probing Nitrosyl Ligation of Surface-Confined Metalloporphyrins by Inelastic Electron Tunneling Spectroscopy. ACS Nano 2013, 7, 5273-5281.

50. Thole, B. T.; Carra, P.; Sette, F.; van der Laan, G. X-ray Circular Dichroism as a Probe of Orbital Magnetization. Phys. Rev. Lett. 1992, 68, 1943-1946.

51. Carra, P.; Thole, B. T.; Altarelli, M.; Wang, X. X-ray Circular Dichroism and Local Magnetic Fields. Phys. Rev. Lett. 1993, 70, 694-697.

52. Stevens, E. D. Electronic Structure of Metalloporphyrins. 1. Experimental Electron Density Distribution of (mesoTetraphenylporphinato)Cobalt(II). J. Am. Chem. Soc. 1981, 103, 5087-5095.

53. Liao, M.-S.; Scheiner, S. Electronic Structure and Bonding in Metal Porphyrins, Metal: $\mathrm{Fe}, \mathrm{Co}, \mathrm{Ni}, \mathrm{Cu}, \mathrm{Zn}$. J. Chem. Phys. 2002, 117, 205.

54. Hermanns, C. F.; Tarafder, K.; Bernien, M.; Krüger, A.; Chang, Y.-M.; Oppeneer, P. M.; Kuch, W. Magnetic Coupling of Porphyrin Molecules through Graphene. Adv. Mater. 2013, 25, 3473-3477.

55. Zhao, A.; Li, Q.; Chen, L.; Xiang, H.; Wang, W.; Pan, S.; Wang, B.; Xiao, X.; Yang, J.; Hou, J. G.; et al. Controlling the Kondo Effect of an Adsorbed Magnetic lon through Its Chemical Bonding. Science 2005, 309, 1542-1544.

56. Stepanow, S.; Miedema, P. S.; Mugarza, A.; Ceballos, G.; Moras, P.; Cezar, J. C.; Carbone, C.; de Groot, F. M. F.; Gambardella, P. Mixed-Valence Behavior and Strong Correlation Effects of Metal Phthalocyanines Adsorbed on Metals. Phys. Rev. B: Condens. Matter Mater. Phys. 2011, 83, 220401

57. Seufert, K.; Bocquet, M.-L.; Auwärter, W.; Weber-Bargioni, A.; Reichert, J.; Lorente, N.; Barth, J. V. cis-Dicarbonyl Binding at Cobalt and Iron Porphyrins with Saddle-Shape Conformation. Nat. Chem. 2011, 3, 114-119.

58. Flechtner, K.; Kretschmann, A.; Steinrück, H.P.; Gottfried, J. M. NO-Induced Reversible Switching of the Electronic Interaction between a Porphyrin-Coordinated Cobalt lon and a Silver Surface. J. Am. Chem. Soc. 2007, 129, 12110-12111.

59. Bohn, R. K.; Haaland, A. On the Molecular Structure of Ferrocene, $\mathrm{Fe}\left(\mathrm{C}_{5} \mathrm{H}_{5}\right)_{2}$. J. Organomet. Chem. 1966, 5, 470-476.

60. Haaland, A. Molecular Structure and Bonding in 3d Metallocenes. Acc. Chem. Res. 1979, 12, 415-422.
61. Stevenson, D. L.; Wei, C. H.; Dahl, L. F. Organometallic Chalcogen Complexes. XXI. Stereochemical Analysis of the Mixed-Metal $\mathrm{FeCO}_{2}(\mathrm{CO})_{9} \mathrm{~S}$ Cluster System and Resulting Bond-Length Evidence for Antibonding Trimetal Character of the Unpaired Electron in the Isostructural $\mathrm{Co}_{3}(\mathrm{CO})_{9} \mathrm{~S}$ Cluster System. J. Am. Chem. Soc. 1971, 93, 6027-6031.

62. Nguyen, T.; Merrill, W. A.; Ni, C.; Lei, H.; Fettinger, J. C.; Ellis, B. D.; Long, G. J.; Brynda, M.; Power, P. P. Synthesis and Characterization of the Metal(I) Dimers [Ar'MMAr']: Comparisons with Quintuple-Bonded $\left[\mathrm{Ar}^{\prime} \mathrm{CrCrAr}^{\prime}\right]$. Angew. Chem., Int. Ed. 2008, 47, 9115-9117.

63. Buchner, F.; Kellner, I.; Steinrück, H.-P.; Marbach, H. Modification of the Growth of Iron on $\mathrm{Ag}(111)$ by Predeposited Organic Monolayers. Z. Phys. Chem. 2009, 223, 131-144.

64. Niemeyer, M.; Hirsch, K.; Zamudio-Bayer, V.; Langenberg, A.; Vogel, M.; Kossick, M.; Ebrecht, C.; Egashira, K.; Terasaki, A.; Möller, T.; et al. Spin Coupling and Orbital Angular Momentum Quenching in Free Iron Clusters. Phys. Rev. Lett. 2012, 108.

65. Gutsev, G. L.; Bauschlicher, C. W. Chemical Bonding, Electron Affinity, and lonization Energies of the Homonuclear 3d Metal Dimers. J. Phys. Chem. A 2003, 107, 47554767.

66. Horcas, I.; Fernández, R.; Rodríguez, J. M.; Colchero, J.; Gómez-Herrero, J.; Baró, A. M. WSXM: A Software for Scanning Probe Microscopy and a Tool for Nanotechnology. Rev. Sci. Instrum. 2007, 78, 013705.

67. Kresse, G.; Hafner, J. Ab Initio Molecular Dynamics for Open-Shell Transition Metals. Phys. Rev. B: Condens. Matter Mater. Phys. 1993, 48, 13115.

68. Perdew, J. P.; Burke, K.; Ernzerhof, M. Generalized Gradient Approximation Made Simple. Phys. Rev. Lett. 1996, 77, 3865.

69. Grimme, S. Semiempirical GGA-Type Density Functional Constructed with a Long-Range Dispersion Correction. J. Comput. Chem. 2006, 27, 1787.

70. Tersoff, J.; Hamann, D. R. Theory and Application for the Scanning Tunneling Microscope. Phys. Rev. Lett. 1983, 50, 1998.

71. Lorente, N. In Handbook of Surface Science; Hasselbrink, E., Lundqvist, B. I., Eds.; North-Holland: Amsterdam, 2008. 